\title{
Heterosis and Combining Ability for Yield in Muskmelon (Cucumis melo L.)
}

\author{
Singh Varinder* and V.K. Vashisht
}

Department of Vegetable Science, Punjab Agricultural University, Ludhiana-141004, India

*Corresponding author

\section{A B S T R A C T}

\begin{tabular}{|c|}
\hline Keywords \\
\hline $\begin{array}{l}\text { Muskmelon, Diallel } \\
\text { analysis, Heterosis, } \\
\text { General combining } \\
\text { ability, Specific } \\
\text { combining ability }\end{array}$ \\
\hline Article Info \\
\hline $\begin{array}{l}\text { Accepted: } \\
\text { 17 July } 2018 \\
\text { Available Online: } \\
10 \text { August } 2018\end{array}$ \\
\hline
\end{tabular}

A study was conducted to assess the extent of heterosis and combining ability of muskmelon in a half diallel mating design. Forty five F1 hybrids and ten inbred lines were evaluated for fourteen different characters during 2014-15 at Department of Vegetable Science, Punjab Agricultural University, Ludhiana. The analysis of variance indicated significant variability among all the genotypes for all the traits except fruit weight and acidity. The combining ability analysis revealed that general combining ability effects and specific combining ability effects were significant for all the traits. Among inbred lines, MS-5 and MM-311 were found to be the best general combiners for days to fruit maturity, fruit yield per plot and TSS content along with other important traits. Cross combination, MM-309 $\times$ MM-311 exhibited highest specific combining ability for total fruit yield per plot, while cross combinations, MS-5 $\times$ MM 2008-8 and MS-5 × MM-308 were best specific combiners for TSS content and days to fruit maturity, respectively. Cross combinations viz., MS-5 × MM 308 and MS-5 $\times$ MM-304 were significantly better for days to fruit maturity, total fruit yield per plot and TSS content along with other important fruit traits over standard checks, Punjab Hybrid and Farmers' Glory.

\section{Introduction}

Muskmelon (Cucumis melo L.) is an important cucurbitaceous crop relished as a dessert fruit for its sweet taste. It has gained commercial importance due to its short duration and high production potential. Heterosis breeding has been the most successful approach among various technological options available to plant breeders for the improvement of productivity in crop plants. This phenomenon though not fully understood genetically so far, has yet enabled the plant scientists to improve the performance of several economic traits
(Chahal and Gosal, 2014). Hybrids have their effect on the cultivation of vegetable crops especially tomato, onion and cole crops. The hybrids in tomato have been released which carry high degree of resistance to nematodes and can tolerate heat. The hybrids are cultivated on extensive scale in crops like cotton and tomato even with the seed produced through the cumbersome and costly process of manual emasculation and pollination, which is a reflection of superior potential of hybrids over traditional varieties. Apart from their per se contribution to increased agricultural production, the hybrid 
varieties led to the establishment of private seed industry engaged in plant breeding research. At present a major part of the basic and applied research on development of hybrids in most of crop plants lies with the private industry. The improvements in breeding methodologies have led not only to intensification of heterosis but also to the development of successful single cross hybrids which occupy practically the entire area under maize in USA (Dhillon, 1998).

In India, private seed companies are marketing hybrids of muskmelon having high TSS and better shelf life along with other superior horticultural traits. Their hybrids are giving stiff competition to public sector hybrids. Therefore, there is an urgent need to develop $\mathrm{F}_{1}$ hybrids possessing high and stable TSS, longer shelf life along with earliness. In order to develop hybrids competitive with hybrids of private seed companies, there is a need to exploit the new inbred lines and elite germplasm collections for selecting potential parents. $F_{1}$ hybrids play an important role in increasing muskmelon production due to their early maturity, high yield potential, superior quality, disease and insect-pest resistance (Banga and Banga, 2000). Further, the development of $F_{1}$ hybrids is the quickest way of improving important economic traits and an easy way of introducing disease resistance governed by dominant genes.

To develop the hybrids with desired traits, choice of the parents is one of the critical and most important tasks for vegetable breeders. The common approach for selecting the parents on the basis of per se performance, does not necessarily lead to good hybrids. The ability of parents to nick well depends on the genes, which cannot be merely adjudged by per se performance of the parents. Diallel mating design proposed by Griffing (1956) is an ideal to produce maximum hybrid combinations from a given number of parents.
Diallel cross design is frequently used in plant breeding research to obtain information on genetic effects for a fixed set of parental lines or to estimate general combining ability (GCA), specific combining ability (SCA), variance components and heritability for a population from randomly chosen parental lines. The main advantage of a half diallel design is that each parent is crossed with each other in all possible combinations (excluding the reciprocals). It requires half matings than full diallel design and requires less experimental area for evaluation of material. It generates information about the performance of parents and their hybrids. It is better method as compared to line $\mathrm{x}$ tester because in the line $\mathrm{x}$ tester design, each parent does not get equal chance to mate with every other parent, whereas in a diallel cross each parent has equal chance to mate with the every other parent (Ferreira et al., 2002).

\section{Materials and Methods}

The present investigations were conducted during 2014- 2015 in the Department of Vegetable Science, Punjab Agricultural University, Ludhiana, India. The experimental materials comprised ten inbred lines and forty five $F_{1}$ hybrids and three standard checks. The crosses were attempted during spring-summer season of 2014. The hermaphrodite flowers were emasculated one day before opening of the flowers in the evening. Both the male and emasculated flowers were covered at bud stage with white parchment bags in the evening prior to anthesis. The emasculated flowers were used as female flowers during crossing. In the next morning, the bags from the freshly opened male flowers and emasculated flowers were removed and emasculated flowers were pollinated with the pollens of the desired freshly opened male flowers. After pollination, each pollinated flower was tagged and again covered with parchment paper bag. Likewise, all the crosses 
were attempted and simultaneously each parent was selfed. Selfing was done by bagging the hermaphrodite flowers in the evening and these were pollinated by taking pollens from covered male flowers of the same plant. The $F_{1}$ seeds of different crosses and selfed seeds of parents were collected during June 2014.Nursery was sown in the mid-Feb 2015 with $F_{1}$ hybrid seeds, parents and check hybrids viz., Punjab Hybrid, MH27 and Farmers' Glory. Two seeds of each entry were sown in each polythene bag and kept under protected cover to save the emerging seedlings from low temperature. When the seedlings attained two to three true leaves stage, one of two seedlings was removed in each polythene bag. Irrigation was withheld for one day prior to transplanting to harden the seedlings. The seedlings were transplanted in the evening in the experimental field by removing the polythene bags. Ten plants of each genotype were transplanted in the mid of March 2015 on edges of raised beds at a distance of $0.60 \mathrm{~m}$ whereas the channels were spaced at $3.0 \mathrm{~m}$. Observations were recorded on eight plants. The experiment was laid out in a Randomized Complete Block Design (RBD) with two replications. The package of practices recommended for the crop was followed to raise a healthy crop (Anon, 2014). The data of individual plants of each progeny were recorded for days taken to first pistillate flower opening, days taken to first fruit maturity, fruit weight $(\mathrm{kg})$, total yield per plot $(\mathrm{kg})$, fruit shape index, fruit cavity area $\left(\mathrm{cm}^{2}\right)$, flesh thickness $(\mathrm{cm})$, total soluble solids content (\%), reaction to fusarium wilt disease, reaction to viral diseases.

\section{Results and Discussion}

The analysis of variance for the experimental design forvarious characters Table 1 . Revealed that the mean squares due to genotypes were significant forall the characters indicating potential genetic differences among genotypes, i.e., parents and their hybrids. The analysis of variance for combining ability for different characters is presented in Table 2. The mean squares due to gca and sca were highly significant for all the characters except days to first pistillate flower opening in gca indicating that both additive and non-additive variances were important in controlling the expression of the traits evaluated. Among ten parents, MS-5 was the best general combiner for four characters viz., days to first pistillate flower opening, days to fruit maturity, fruit weight and total yield per plot (Table 3). However, MM-311 was the best general combiner for three characters viz., flesh thickness, fruit cavity area and ascorbic acid. Similarly, MM-310 was the best general combiner for rind thickness. On the other hand MM-303 was the best general combiner for $\beta$ carotene content and Punjab Sunehri was best general combiner for acidity. Similarly MMMM-304 was the best combiner for TSS content. However, IC-267375 was the best combiner for reaction to fusarium wilt disease. For viral diseases the best general combiner was MM-303. Choudhary et al., (2003) reported that parental line DMDR-1 had a good combining ability for fruit yield per plant, number of primary branches, number of fruits per plant, fruit weight, moisture content, total soluble solids, acidity and total soluble sugars. Vashisht et al., (2010) documented that parental line Hara Madhu was the best general combiner for total fruit yield per vine (0.17), fruit weight (0.05), number of fruits per vine (0.11), TSS content (0.52) and fruit shape index (0.045) while Punjab Rasila was the best general combiner for fruit/cavity ratio (0.16), flesh thickness (0.105), flesh proportion $(0.018)$ and node at which $1^{\text {st }}$ female flower opens $(-0.26)$.

It was observed that the cross combination IC$267375 \times$ MM-303 had highest sca values in significant direction for days to first pistillate 
flower and MM-310 $\times$ MM-304 for days to maturity (Table 4). For TSS content, best cross combination was MS-5 × MM 2008-8. The cross combination MS-5 $\times$ MM-310 had highest significant value for fruit weight. Cross combination MS-5 × MM-304 had the significant value for flesh thickness. Cross combination MM-303 $\times$ Punjab Sunehri had highest significant sca value for rind thickness. In case of total yield per plot, the best cross combination was mm-2008-8 $\times$ MM-311. Cross combination Punjab Sunehri $\times$ MM-304 had significant values for area of fruit cavity. However, cross combinations, MM 2008-8 × Punjab Sunehri, MM 2008-8 × MM-303, IC-267375 × MM-304 were found to be best for ascorbic acid content, acidity and beta carotene content, respectively. Cross combination MM-303 × Punjab Sunehri had highest significant sca value for reaction to fusarium wilt diseases. Cross combination MS-5 × MM-306 had significant effect for reaction to viral diseases.

In case of fruit weight some studies have been reported by Munshi and Verma (1997), Gurav et al., (2000) and Vashisht et al., (2010). Whereas, for TSS content sca effects were reported by Liou et al., (1995), Dhaliwal and Lal (1996). Higher sca effects for earliness were suggested by Kumar et al., (2005a). In case of fruit yield, significant sca effects were recorded by various workers (Gurav et al., (2000), Moon et al., (2003), Tomar and Bhalala (2006a), Tomar and Bhalala (2006b), Glala et al., (2011)

Table.1 Analysis of variance for design of experiment for different characters of muskmelon

\begin{tabular}{|l|c|c|c|}
\hline \multicolumn{1}{|c|}{ Source of variation } & Replications & Genotypes & Error \\
\hline df & 1 & 54 & 54 \\
\hline Days to first pistillate opening & 1.12 & $4.39 * *$ & 0.3 \\
\hline Days to fruit maturity & 0.87 & $7.17 * *$ & 0.33 \\
\hline Fruit weight (kg) & 0 & 0.03 & 0 \\
\hline TSS content (\%) & 1.37 & $4.40 * *$ & 0.27 \\
\hline Rind thickness (mm) & 0.01 & $1.59 * *$ & 0.07 \\
\hline Flesh thickness(cm) & 0.04 & $0.30 *$ & 0.02 \\
\hline Fruit cavity area (cm $\left.\mathbf{c m}^{\mathbf{2}}\right)$ & 3.99 & $211.87 * *$ & 2.31 \\
\hline Total fruit yield per plot & 0 & $35.03 * *$ & 3.68 \\
\hline
\end{tabular}

Table.2 Analysis of variance for combining ability for different characters of muskmelon

\begin{tabular}{|l|}
\hline Source of variation \\
\hline df \\
\hline Days to first pistillate flower opening \\
\hline Days to fruit maturity \\
\hline Fruit weight $(\mathrm{kg})$ \\
\hline TSS content $(\%)$ \\
\hline Rind thickness $(\mathrm{mm})$ \\
\hline Flesh thickness $(\mathrm{cm})$ \\
\hline Fruit cavity area $\left(\mathrm{cm}^{2}\right)$ \\
\hline Total fruit yield per plot $(\mathrm{kg})$ \\
\hline
\end{tabular}

\begin{tabular}{|c|c|c|}
\hline GCA & SCA & Error \\
\hline 9 & 45 & 54 \\
\hline 5.86 & $1.47 * *$ & 0.15 \\
\hline $9.25 * *$ & $2.44 * *$ & 0.17 \\
\hline $0.02 * *$ & $0.01 * *$ & 0 \\
\hline $4.82 * *$ & $1.68 * *$ & 0.13 \\
\hline $2.66 * *$ & $0.43 * *$ & 0.17 \\
\hline $0.32 * *$ & $0.11 * *$ & 0.01 \\
\hline $213.01 * *$ & $84.52 * *$ & 1.16 \\
\hline $42.95 * *$ & $12.43 * *$ & 1.84 \\
\hline
\end{tabular}


Table.3 Estimation of gca effects of parents for different characters of muskmelon

\begin{tabular}{|c|c|c|c|c|c|c|c|c|}
\hline Parents & $\begin{array}{c}\text { Days to } \\
\text { first } \\
\text { pistillate } \\
\text { opening }\end{array}$ & $\begin{array}{c}\text { Days to } \\
\text { fruit } \\
\text { maturity }\end{array}$ & $\begin{array}{c}\text { Fruit } \\
\text { weight } \\
\text { (kg) }\end{array}$ & $\begin{array}{c}\text { TSS } \\
\text { content } \\
(\%)\end{array}$ & $\begin{array}{c}\text { Rind } \\
\text { thicknes } \\
\text { s (mm) }\end{array}$ & $\begin{array}{c}\text { Flesh } \\
\text { thicknes } \\
\text { S } \\
(\mathrm{cm})\end{array}$ & $\begin{array}{c}\text { Fruit } \\
\text { cavity } \\
\text { area } \\
\left(\mathrm{cm}^{2}\right)\end{array}$ & $\begin{array}{c}\text { Total } \\
\text { fruit } \\
\text { yield per } \\
\text { plot } \\
\text { (kg) }\end{array}$ \\
\hline MS-5 & $-1.201 * *$ & $-1.585^{* *}$ & $0.077 * *$ & 0.150 & $-0.112 *$ & $-0.286^{* *}$ & $6.812 * *$ & $3.475^{* *}$ \\
\hline MM 2008-8 & 0.045 & $1.769 * *$ & $-0.055^{* *}$ & $-0.989 * *$ & $-1.166 * *$ & $-0.098 * *$ & $-2.430 * *$ & $1.350 * *$ \\
\hline MM-310 & -0.168 & 0.207 & $-0.020 * *$ & $-0.673 * *$ & $0.597 * *$ & $-0.198 * *$ & $-4.084 * *$ & -0.379 \\
\hline IC-267375 & $0.362 * *$ & -0.177 & $0.013^{*}$ & $-0.889 * *$ & $0.272 * *$ & -0.007 & $3.703 * *$ & $-0.838 *$ \\
\hline MM-303 & $0.983 * *$ & $0.269 *$ & $-0.024 * *$ & $0.352 * *$ & $0.351 * *$ & 0.018 & $4.435 * *$ & $-1.088 * *$ \\
\hline MM-308 & $-0.518 * *$ & $0.478 * *$ & 0.010 & $0.504 * *$ & $-0.170 * *$ & 0.014 & $-0.722 *$ & 0.642 \\
\hline PS & $-0.738 * *$ & $-0.814 * *$ & $-0.021 * *$ & -0.098 & -0.078 & 0.002 & -1.599 & -0.546 \\
\hline MM-306 & $0.766^{* *}$ & -0.206 & 0.003 & $0.373 * *$ & 0.076 & $0.118 * *$ & $2.941 * *$ & $-2.504 * *$ \\
\hline MM-311 & $0.628 * *$ & $0.353 * *$ & $0.033 * *$ & $0.410 * *$ & 0.105 & $0.243 * *$ & $-6.055^{* *}$ & $-2.213 * *$ \\
\hline MM-304 & -0.159 & $-0.293 *$ & $-0.017 * *$ & $0.860 * *$ & $0.126^{*}$ & $0.193 * *$ & $-3.001 * *$ & $2.100 * *$ \\
\hline CD 5\% & 0.213 & 0.224 & 0.012 & 0.201 & 0.106 & 0.050 & 0.590 & 0.744 \\
\hline CD $1 \%$ & 0.284 & 0.298 & 0.017 & 0.267 & 0.141 & 0.067 & 0.786 & 0.991 \\
\hline
\end{tabular}

Table.4 Estimation of sca effects of cross combinations for different characters of muskmelon

\begin{tabular}{|c|c|c|c|c|c|c|c|c|}
\hline Hybrids & $\begin{array}{l}\text { Days to first } \\
\text { pistillate } \\
\text { opening }\end{array}$ & $\begin{array}{l}\text { Days to fruit } \\
\text { maturity }\end{array}$ & $\begin{array}{c}\text { Fruit weight } \\
\text { (kg) }\end{array}$ & $\begin{array}{c}\text { TSS content } \\
(\%)\end{array}$ & $\begin{array}{l}\text { Rind } \\
\text { thickness } \\
(\mathbf{m m})\end{array}$ & $\begin{array}{c}\text { Flesh } \\
\text { thickness }(\mathrm{cm})\end{array}$ & $\begin{array}{c}\text { Fruit cavity } \\
\text { area }\left(\mathrm{cm}^{2}\right)\end{array}$ & $\begin{array}{l}\text { Total fruit } \\
\text { yield per } \\
\text { plot(kg) }\end{array}$ \\
\hline MS-5 X MM 2008-8 & 0.091 & $1.135 * *$ & 0.007 & $2.033 * *$ & $0.474 * *$ & $0.368 * *$ & $23.286 * *$ & 0.511 \\
\hline MS-5 X MM-310 & $-0.696^{*}$ & $1.697 * *$ & $0.197 * *$ & $1.220 * *$ & 0.111 & $-0.932 * *$ & -0.660 & 0.741 \\
\hline MS-5 X IC-267375 & $0.775^{*}$ & $-1.170 * *$ & 0.020 & 0.433 & -0.014 & $-0.224 * *$ & $-8.948 * *$ & $-5.551 * *$ \\
\hline MS-5 X MM-303 & 0.154 & 0.135 & $0.091 * *$ & $0.941 * *$ & $0.557 * *$ & $0.351 * *$ & $17.621 * *$ & $2.949 *$ \\
\hline MS-5 X MM-308 & $1.154 * *$ & $-1.086 * *$ & $0.112 * *$ & $0.914 * *$ & 0.078 & $0.255^{* *}$ & $-6.323 * *$ & $4.220 * *$ \\
\hline MS-5 X PS & 0.625 & $-1.782 * *$ & $-0.072 * *$ & 0.141 & -0.264 & -0.082 & $-5.296 * *$ & $-2.843^{*}$ \\
\hline MS-5 X MM-306 & $-2.130 * *$ & -0.153 & 0.001 & 0.170 & $0.532 * *$ & $-0.249 * *$ & $5.165 * *$ & $4.366 * *$ \\
\hline MS-5 X MM-311 & $-1.242 * *$ & $-0.949 * *$ & 0.014 & $-2.368 * *$ & $-0.697 * *$ & -0.024 & 1.511 & -1.926 \\
\hline MS-5 X MM-304 & -0.405 & $2.197 * *$ & $-0.086^{* *}$ & $0.933 * *$ & $0.782 * *$ & $0.526 * *$ & $6.256 * *$ & 1.761 \\
\hline MM 2008-8 X MM-310 & 0.308 & $1.093 * *$ & $0.063 * *$ & $1.356 * *$ & $-0.935 * *$ & -0.070 & $-2.069 *$ & 1.366 \\
\hline MM 2008-8 X IC-267375 & 0.029 & -0.274 & 0.016 & $1.072 * *$ & -0.010 & 0.039 & -0.606 & 0.324 \\
\hline
\end{tabular}




\begin{tabular}{|c|c|c|c|c|c|c|c|c|}
\hline MM 2008-8 X MM-303 & 0.158 & $0.780^{*}$ & $0.117 * *$ & $-1.671 * *$ & $-0.739 * *$ & $-0.186^{*}$ & $12.337 * *$ & $-4.426 * *$ \\
\hline MM 2008-8 X MM-308 & 0.408 & 0.560 & -0.011 & $-1.822 * *$ & $-0.518 * *$ & $-0.482 * *$ & $-4.181 * *$ & $-2.655^{*}$ \\
\hline MM 2008-8 X PS & $1.129 * *$ & -0.136 & $0.090 * *$ & $-2.469 * *$ & 0.241 & $-0.220 * *$ & -0.004 & 1.032 \\
\hline MM 2008-8 X MM-306 & $-1.375 * *$ & $-1.007 * *$ & $-0.184 * *$ & 0.559 & $-0.414 *$ & $0.164 *$ & $-5.344 * *$ & $-2.510 *$ \\
\hline MM 2008-8 X MM-311 & 0.512 & -0.303 & $-0.124 * *$ & $-1.228 * *$ & $-0.693 * *$ & $-0.261 * *$ & $4.902 * *$ & $8.699 * *$ \\
\hline MM 2008-8 X MM-304 & 0.050 & 0.093 & -0.010 & -0.178 & $0.336^{*}$ & -0.011 & $8.098 * *$ & 0.386 \\
\hline MM-310 X IC-267375 & -0.088 & -0.211 & $-0.124 * *$ & $-1.495 * *$ & $0.928 * *$ & $0.489 * *$ & $4.298 * *$ & $7.553 * *$ \\
\hline MM-310 X MM-303 & $-1.380 * *$ & 0.343 & $-0.053 * *$ & $-0.987 * *$ & $0.599 * *$ & $0.364 * *$ & $-4.683 * *$ & 1.803 \\
\hline MM-310 X MM-308 & 0.121 & 0.122 & $0.038^{*}$ & $-1.638 * *$ & 0.020 & $-0.282 * *$ & 0.473 & 1.074 \\
\hline MM-310 X PS & $-1.659 * *$ & $-1.574 * *$ & $0.175^{* *}$ & 0.464 & 0.128 & $-0.270 * *$ & 0.350 & 1.761 \\
\hline MM-310 X MM-306 & $-0.663^{*}$ & -0.445 & $0.056 * *$ & $-1.107 * *$ & -0.226 & 0.114 & -0.439 & 0.220 \\
\hline MM-310 X MM-311 & $0.975 * *$ & 0.260 & $-0.079 * *$ & -0.394 & $0.345^{*}$ & $-0.511 * *$ & $10.806 * *$ & $3.178 * *$ \\
\hline MM-310 X MM-304 & $0.762 *$ & $-3.095 * *$ & $-0.060 * *$ & $-0.995 * *$ & 0.224 & 0.139 & $9.252 * *$ & $-2.885^{*}$ \\
\hline IC-267375 X MM-303 & $-2.159 * *$ & -0.524 & $0.040 *$ & -0.193 & $-0.726 * *$ & -0.028 & $-4.471 * *$ & -1.239 \\
\hline IC-267375 X MM-308 & $1.591 * *$ & $1.255^{* *}$ & $-0.094 * *$ & $-1.071 * *$ & $0.695^{* *}$ & 0.076 & $3.086 * *$ & 0.532 \\
\hline IC-267375 X PS & 0.312 & $2.810 * *$ & 0.033 & $-1.069 * *$ & $-0.497 * *$ & 0.089 & $9.563 * *$ & 0.220 \\
\hline IC-267375 X MM-306 & $-0.942 * *$ & $0.939 * *$ & $-0.071 * *$ & $-0.790 *$ & $-1.451 * *$ & $-0.328 * *$ & $-7.477 * *$ & $-3.322 * *$ \\
\hline IC-267375 X MM-311 & -0.555 & $-1.857 * *$ & $-0.066^{* *}$ & $-0.777^{*}$ & -0.230 & 0.047 & -1.481 & $-2.614^{*}$ \\
\hline IC-267375 X MM-304 & $-1.767 * *$ & $-2.711 * *$ & $-0.072 * *$ & $-1.528 * *$ & $-1.201 * *$ & $-0.553 * *$ & $-2.785^{* *}$ & -0.926 \\
\hline MM-303 X MM-308 & $0.971 * *$ & $-1.441 * *$ & $-0.108 * *$ & $0.687 *$ & 0.016 & 0.051 & $26.454 * *$ & 1.282 \\
\hline MM-303 X PS & $1.191 * *$ & $2.114 * *$ & $-0.101 * *$ & $-1.561 * *$ & $1.024 * *$ & -0.136 & $-11.044 * *$ & -1.530 \\
\hline MM-303 X MM-306 & $3.687 * *$ & 0.493 & $-0.085^{* *}$ & $1.218 * *$ & $0.370^{*}$ & $0.197 *$ & $-15.208 * *$ & $-3.822 * *$ \\
\hline MM-303 X MM-311 & 0.325 & $-0.803^{*}$ & $-0.180 * *$ & $1.680 * *$ & $0.341^{*}$ & -0.028 & $1.288 * *$ & $-2.864 *$ \\
\hline MM-303 X MM-304 & 0.612 & $1.843 * *$ & -0.026 & $0.730 *$ & 0.170 & $0.222 * *$ & $-4.017 * *$ & $-3.176 * *$ \\
\hline MM-308 X PS & -0.309 & $1.143 * *$ & $0.040 *$ & 0.287 & -0.005 & $0.368 * *$ & -1.762 & 1.741 \\
\hline MM-308 X MM-306 & $-1.813 * *$ & $-3.078 * *$ & $-0.094 * *$ & 0.217 & -0.110 & $-0.449 * *$ & $-4.302 * *$ & $-4.301 * *$ \\
\hline MM-308 X MM-311 & -0.175 & $-2.024 * *$ & $-0.129 * *$ & 0.279 & -0.239 & $-0.324 * *$ & 1.194 & 1.907 \\
\hline MM-308 X MM-304 & 0.112 & -0.628 & $0.050 * *$ & 0.078 & -0.210 & -0.124 & $-4.860 * *$ & -1.905 \\
\hline PSX MM-306 & -0.242 & -0.674 & $-0.053 * *$ & -0.581 & $0.799 * *$ & $0.164^{*}$ & $12.825 * *$ & 0.886 \\
\hline PS X MM-311 & $-1.205^{* *}$ & $-0.720 *$ & $0.147 * *$ & $0.781 *$ & 0.020 & -0.111 & $-4.679 * *$ & $-3.405 * *$ \\
\hline PS X MM-304 & $-0.667 *$ & $-1.074 * *$ & $0.067 * *$ & $0.931 * *$ & $0.349 *$ & 0.039 & $-1.983 *$ & -0.718 \\
\hline MM-306 X MM-311 & $1.041 * *$ & 0.410 & 0.028 & $0.809 * *$ & -0.285 & -0.128 & $-2.719 * *$ & 2.053 \\
\hline MM-306 X MM-304 & $0.829 *$ & -0.195 & 0.028 & $1.219 * *$ & -0.205 & -0.078 & $-7.773 * *$ & $-3.260 * *$ \\
\hline MM-311 X MM-304 & $-1.033 * *$ & -0.241 & 0.013 & -0.228 & 0.266 & 0.047 & 1.723 & 1.449 \\
\hline CD $5 \%$ & 0.643 & 0.675 & 0.038 & 0.605 & 0.320 & 0.152 & 1.778 & 2.243 \\
\hline CD $1 \%$ & 0.857 & 0.899 & 0.050 & 0.806 & 0.426 & 0.202 & 2.369 & 2.989 \\
\hline
\end{tabular}


Table.5 Estimation of heterosis (\%) over commercial checks, Punjab Hybrid, Farmers' Glory and MH-27 for different characters

\begin{tabular}{|c|c|c|c|c|c|c|c|c|c|}
\hline \multirow[t]{2}{*}{ Hybrid } & \multicolumn{3}{|c|}{ Days to first pistillate opening } & \multicolumn{3}{|c|}{ Days to fruit maturity } & \multicolumn{3}{|c|}{ Fruit weight (kg) } \\
\hline & PH & FG & MH-27 & PH & FG & MH-27 & PH & FG & MH-27 \\
\hline MS-5 X MM 2008-8 & $2.22^{* *}$ & $6.98 * *$ & 0.00 & 0.73 & $6.15 * *$ & -0.72 & $13.79 * *$ & $-11.29 * *$ & $-15.38^{* *}$ \\
\hline MS-5 X MM-310 & $-2.22 * *$ & $2.33 * *$ & $-4.35^{* * *}$ & -0.73 & $4.62 * *$ & $-2.16 * *$ & $44.83 * *$ & $12.90 * *$ & $7.69 * *$ \\
\hline MS-5 X IC-267375 & $6.67 * *$ & $11.63^{* *}$ & $4.35 * *$ & $-5.47 * *$ & -0.38 & $-6.83^{* *}$ & $24.83^{* *}$ & $-2.69 * *$ & $-7.18 * *$ \\
\hline MS-5 X MM-303 & $6.67 * *$ & $11.63^{* *}$ & $4.35^{* *}$ & $-2.92 * *$ & $2.31 * *$ & $-4.32 * *$ & $29.66^{* *}$ & 1.08 & $-3.59 * *$ \\
\hline MS-5 X MM-308 & $4.44 * *$ & $9.30 * *$ & $2.17 * *$ & $-4.38 * *$ & 0.77 & $-5.76 * *$ & $37.24 * *$ & $6.99 * *$ & $2.05^{*}$ \\
\hline MS-5 X PS & $1.11^{*}$ & $5.81 * *$ & $-1.09 *$ & $-7.30 * *$ & $-2.31^{* *}$ & $-8.63 * *$ & $7.59^{* *}$ & $-16.13^{* * *}$ & $-20.00^{* *}$ \\
\hline MS-5 X MM-306 & $-4.44 * *$ & $0.00 * *$ & $-6.52^{* * *}$ & $-4.01 * *$ & $1.15^{*}$ & -5.40 ** & $20.69 * *$ & $-5.91 * *$ & $-10.26^{* * *}$ \\
\hline MS-5 X MM-311 & $-1.11^{*}$ & $3.49 * *$ & $-3.26 * *$ & $-4.38 * *$ & 0.77 & $-5.76^{* * *}$ & $26.90^{* *}$ & -1.08 & $-5.64 * *$ \\
\hline MS-5 X MM-304 & -0.89 & $3.72 * *$ & $-3.04 * *$ & -0.73 & $4.62^{* * *}$ & $-2.16^{* *}$ & $6.21 * *$ & $-17.20^{* *}$ & $-21.03 * *$ \\
\hline MM 2008-8 X MM-310 & $7.78^{* * *}$ & $12.79 * *$ & $5.43 * *$ & $3.28 * *$ & $8.85 * *$ & $1.80^{* * *}$ & $8.28 * *$ & $-15.59^{* * *}$ & $-19.49 * *$ \\
\hline MM 2008-8 X IC-267375 & $8.89^{* *}$ & $13.95 * *$ & $6.52 * *$ & 0.73 & $6.15 * *$ & -0.72 & $6.21 * *$ & $-17.20^{* * *}$ & $-21.03 * *$ \\
\hline MM 2008-8 X MM-303 & $12.22 * *$ & $17.44^{* *}$ & $9.78^{* *}$ & $2.92 * *$ & $8.46 * *$ & $1.44 *$ & $15.17^{* *}$ & $-10.22 * *$ & $-14.36^{* *}$ \\
\hline MM 2008-8 X MM-308 & $6.67 * *$ & $11.63^{* *}$ & $4.35 * *$ & $2.92^{* *}$ & $8.46^{* * *}$ & $1.44 *$ & $2.07 *$ & $-20.43^{* *}$ & $-24.10^{* * *}$ \\
\hline MM 2008-8 X PS & $8.89^{* *}$ & $13.95 * *$ & $6.52^{* *}$ & 0.00 & $5.38^{* *}$ & $-1.44 *$ & $11.72 * *$ & $-12.90^{* * *}$ & $-16.92^{* *}$ \\
\hline MM 2008-8 X MM-306 & $4.44^{* *}$ & $9.30^{* *}$ & $2.17 * *$ & -0.36 & $5.00^{* * *}$ & $-1.80^{* *}$ & $-22.76^{* * *}$ & $-39.78^{* * *}$ & $-42.56^{* *}$ \\
\hline MM 2008-8 X MM-311 & $12.22 * *$ & $17.44^{* *}$ & $9.78^{* *}$ & $1.46^{*}$ & $6.92 * *$ & 0.00 & $-10.34 * *$ & $-30.11^{* * *}$ & $-33.33^{* * *}$ \\
\hline MM 2008-8 X MM-304 & $6.67 * *$ & $11.63^{* *}$ & $4.35 * *$ & 1.09 & $6.54 * *$ & -0.36 & -1.38 & $-23.12^{* *}$ & $-26.67 * *$ \\
\hline MM-310 X IC-267375 & $7.78^{* *}$ & $12.79 * *$ & $5.43 * *$ & $-1.46 *$ & $3.85 * *$ & $-2.88 * *$ & $-8.28 * *$ & $-28.49 * *$ & $-31.79 * *$ \\
\hline MM-310 X MM-303 & $4.44 * *$ & $9.30 * *$ & $2.17 * *$ & 0.00 & $5.38 * *$ & $-1.44 *$ & $-3.45 * *$ & $-24.73^{* *}$ & $-28.21 * *$ \\
\hline MM-310 X MM-308 & $4.44 * *$ & $9.30^{* *}$ & $2.17 * *$ & 0.00 & $5.38^{* * *}$ & $-1.44 *$ & $13.79 * *$ & $-11.29 * *$ & $-15.38^{* * *}$ \\
\hline MM-310 X PS & $-4.44 * *$ & $0.00 * *$ & $-6.52^{* * *}$ & $-4.38^{* *}$ & 0.77 & $-5.76^{* *}$ & $28.28^{* *}$ & 0.00 & $-4.62 * *$ \\
\hline MM-310 X MM-306 & $6.67 * *$ & $11.63^{* *}$ & $4.35^{* * *}$ & $-1.82 * *$ & $3.46 * *$ & $-3.24 * *$ & $15.17 * *$ & $-10.22^{* * *}$ & $-14.36^{* * *}$ \\
\hline MM-310 X MM-311 & $13.33^{* *}$ & $18.60^{* *}$ & $10.87^{* *}$ & 0.00 & $5.38^{* * *}$ & $-1.44 *$ & 0.69 & $-21.51^{* *}$ & $-25.13 * *$ \\
\hline MM-310 X MM-304 & $8.89 * *$ & $13.95 * *$ & $6.52^{* * *}$ & $-5.84 * *$ & -0.77 & $-7.19^{* * *}$ & $-3.45^{* *}$ & $-24.73^{* * *}$ & $-28.21 * *$ \\
\hline IC-267375 X MM-303 & $3.33^{* *}$ & $8.14 * *$ & $1.09 *$ & $-1.82^{* *}$ & $3.46^{* * *}$ & $-3.24 * *$ & $13.79 * *$ & $-11.29 * *$ & $-15.38^{* * *}$ \\
\hline IC-267375 X MM-308 & $13.33^{* *}$ & $18.60^{* *}$ & $10.87 * *$ & 1.09 & $6.54 * *$ & -0.36 & 0.00 & $-22.04 * *$ & $-25.64 * *$ \\
\hline IC-267375 X PS & $6.67 * *$ & $11.63^{* *}$ & $4.35 * *$ & $1.46^{*}$ & $6.92^{* * *}$ & 0.00 & $13.10^{* *}$ & $-11.83^{* * *}$ & $-15.90^{* * *}$ \\
\hline IC-267375 X MM-306 & $7.78^{* *}$ & $12.79 * *$ & $5.43 * *$ & -0.36 & $5.00^{* *}$ & $-1.80^{* *}$ & $2.07 *$ & $-20.43^{* * *}$ & $-24.10^{* *}$ \\
\hline IC-267375 X MM-311 & $8.89^{* *}$ & $13.95^{* *}$ & $6.52^{* *}$ & $-3.65^{* *}$ & $1.54 * *$ & $-5.04 * *$ & $6.90^{* *}$ & $-16.67 * *$ & $-20.51^{* *}$ \\
\hline IC-267375 X MM-304 & 0.00 & $4.65 * *$ & $-2.17^{* * *}$ & $-5.84 * *$ & -0.77 & $-7.19^{* * *}$ & -0.69 & $-22.58^{* * *}$ & $-26.15^{* * *}$ \\
\hline MM-303 X MM-308 & $13.33^{* *}$ & $18.60^{* *}$ & $10.87^{* * *}$ & $-2.19 * *$ & $3.08 * *$ & $-3.60 * *$ & $-6.90 * *$ & $-27.42^{* * *}$ & $-30.77^{* *}$ \\
\hline MM-303 X PS & $13.33^{* * *}$ & $18.60^{* *}$ & $10.87^{* *}$ & 1.09 & $6.54 * *$ & -0.36 & $-10.34^{* *}$ & $-30.11^{* *}$ & $-33.33^{* *}$ \\
\hline MM-303 X MM-306 & $31.11 * *$ & $37.21 * *$ & $28.26 * *$ & -0.36 & $5.00 * *$ & $-1.80 * *$ & $-4.83^{* *}$ & $-25.81 * *$ & $-29.23 * *$ \\
\hline MM-303 X MM-311 & $15.56^{* *}$ & $20.93 * *$ & $13.04 * *$ & $-1.46^{*}$ & $3.85 * *$ & $-2.88 * *$ & -13.79 ** & $-32.80^{* * *}$ & $-35.90^{* * *}$ \\
\hline MM-303 X MM-304 & $13.33 * *$ & $18.60^{* *}$ & $10.87 * *$ & $1.46^{*}$ & $6.92 * *$ & 0.00 & 0.69 & $-21.51 * *$ & $-25.13^{* * *}$ \\
\hline MM-308 X PS & 0.00 & $4.65 * *$ & $-2.17^{* *}$ & 0.00 & $5.38 * *$ & $-1.44 *$ & $13.79 * *$ & $-11.29 * *$ & $-15.38^{* * *}$ \\
\hline MM-308 X MM-306 & 0.00 & $4.65 * *$ & $-2.17 * *$ & $-5.47 * *$ & -0.38 & $-6.83^{* *}$ & -1.38 & $-23.12^{* * *}$ & $-26.67^{* *}$ \\
\hline MM-308 X MM-311 & $6.67 * *$ & $11.63^{* *}$ & $4.35 * *$ & $-2.92 * *$ & $2.31 * *$ & $-4.32 * *$ & $-2.07^{*}$ & $-23.66 * *$ & $-27.18^{* * *}$ \\
\hline MM-308 X MM-304 & $4.44 * *$ & $9.30 * *$ & $2.17 * *$ & $-1.82^{* *}$ & $3.46 * *$ & $-3.24 * *$ & $15.86^{* *}$ & $-9.68 * *$ & $-13.85^{* *}$ \\
\hline PSX MM-306 & $6.00^{* * *}$ & $10.93 * *$ & $3.70^{* * *}$ & $-3.65 * *$ & $1.54 * *$ & $-5.04 * *$ & 0.00 & $-22.04 * *$ & $-25.64 * *$ \\
\hline PS X MM-311 & $1.11 *$ & $5.81 * *$ & $-1.09 *$ & $-2.92 * *$ & 2.31 ** & $-4.32^{* * *}$ & $31.72 * *$ & $2.69 * *$ & $-2.05^{*}$ \\
\hline PS X MM-304 & 0.00 & $4.65 * *$ & $-2.17 * *$ & $-4.38 * *$ & 0.77 & $-5.76 * *$ & $13.79 * *$ & $-11.29 * *$ & $-15.38 * *$ \\
\hline MM-306 X MM-311 & $17.78^{* *}$ & $23.26^{* *}$ & $15.22^{* *}$ & -0.36 & $5.00^{* * *}$ & $-1.80^{* *}$ & $18.62 * *$ & $-7.53^{* *}$ & $-11.79 * *$ \\
\hline MM-306 X MM-304 & $13.33^{* *}$ & $18.60^{* * *}$ & $10.87 * *$ & $-2.19^{* *}$ & $3.08^{* *}$ & $-3.60 * *$ & $11.72 * *$ & $-12.90^{* *}$ & $-16.92 * *$ \\
\hline MM-311 X MM-304 & $4.44^{* *}$ & $9.30 * *$ & $2.17 * *$ & $-1.46^{*}$ & $3.85 * *$ & $-2.88 * *$ & $13.79 * *$ & $-11.29^{* *}$ & $-15.38^{* *}$ \\
\hline CD 5\% & & 1.07 & & & 1.12 & & & 1.96 & \\
\hline CD 1\% & & 1.41 & & & 1.48 & & & 2.57 & \\
\hline
\end{tabular}




\begin{tabular}{|c|c|c|c|c|c|c|c|c|c|}
\hline \multirow[t]{2}{*}{ Hybrid } & \multicolumn{3}{|c|}{ TSS content (\%) } & \multicolumn{3}{|c|}{ Rind thickness $(\mathrm{mm})$} & \multicolumn{3}{|c|}{ Flesh thickness(cm) } \\
\hline & PH & FG & MH-27 & PH & FG & MH-27 & PH & FG & MH-27 \\
\hline MS-5 X MM 2008-8 & $6.67 * *$ & $6.67 * *$ & $-6.98 * *$ & $11.63^{* *}$ & $-29.41 * *$ & $-37.66^{* *}$ & $21.95 * *$ & $-5.66 * *$ & $-26.47 * *$ \\
\hline MS-5 X MM-310 & $2.22 * *$ & $2.22 * *$ & $-10.85 * *$ & $76.74^{* * *}$ & $11.76^{* *}$ & $-1.30^{* *}$ & $-46.34 * *$ & $-58.49 * *$ & $-67.65^{* *}$ \\
\hline MS-5 X IC-267375 & $-6.67 * *$ & $-6.67 * *$ & $-18.60 * *$ & $55.81^{* *}$ & $-1.47 * *$ & $-12.99 * *$ & $-2.44 * *$ & $-24.53 * *$ & $-41.18 * *$ \\
\hline MS-5 X MM-303 & $8.89 * *$ & $8.89^{* *}$ & $-5.04 * *$ & $86.05^{* *}$ & $17.65^{* *}$ & $3.90^{* *}$ & $26.83^{* *}$ & $-1.89 * *$ & $-23.53^{* *}$ \\
\hline MS-5 X MM-308 & $10.00^{* *}$ & $10.00^{* *}$ & $-4.07 * *$ & $39.53^{* *}$ & $-11.76^{* *}$ & $-22.08 * *$ & $21.95 * *$ & $-5.66^{* *}$ & $-26.47 * *$ \\
\hline MS-5 X PS & $-2.22 * *$ & $-2.22 * *$ & $-14.73 * *$ & $27.91^{* *}$ & $-19.12 * *$ & $-28.57 * *$ & $4.88 * *$ & $-18.87 * *$ & $-36.76^{* *}$ \\
\hline MS-5 X MM-306 & $2.22 * *$ & $2.22 * *$ & $-10.85 * *$ & $72.09 * *$ & $8.82 * *$ & $-3.90 * *$ & $2.44 * *$ & $-20.75^{* *}$ & $-38.24 * *$ \\
\hline MS-5 X MM-311 & $-20.00^{* * *}$ & $-20.00 * *$ & $-30.23 * *$ & $16.28^{* * *}$ & $-26.47 * *$ & $-35.06 * *$ & $19.51 * *$ & $-7.55 * *$ & $-27.94 * *$ \\
\hline MS-5 X MM-304 & $13.33^{* *}$ & $13.33^{* *}$ & $-1.16^{*}$ & $86.05^{* *}$ & $17.65^{* * *}$ & $3.90^{* *}$ & $43.90 * *$ & $11.32 * *$ & $-13.24 * *$ \\
\hline MM 2008-8 X MM-310 & $-6.67 * *$ & $-6.67 * *$ & $-18.60 * *$ & $-20.93 * *$ & $-50.00 * *$ & $-55.84 * *$ & $4.88 * *$ & $-18.87 * *$ & $-36.76^{* *}$ \\
\hline MM 2008-8 X IC-267375 & $-11.11 * *$ & $-11.11^{* *}$ & $-22.48 * *$ & $6.98^{* *}$ & $-32.35 * *$ & $-40.26 * *$ & $19.51 * *$ & $-7.55^{* *}$ & $-27.94 * *$ \\
\hline MM 2008-8 X MM-303 & $-24.44 * *$ & $-24.44^{* *}$ & $-34.11 * *$ & $-23.26 * *$ & $-51.47 * *$ & $-57.14 * *$ & $9.76^{* *}$ & $-15.09 * *$ & -33.82 ** \\
\hline MM 2008-8 X MM-308 & $-24.44 * *$ & $-24.44 * *$ & $-34.11^{* *}$ & $-37.21 * *$ & $-60.29 * *$ & $-64.94 * *$ & $-4.88 * *$ & $-26.42^{* * *}$ & $-42.65^{* *}$ \\
\hline MM 2008-8 X PS & $-35.56 * *$ & $-35.56^{* *}$ & $-43.80 * *$ & $2.33^{* *}$ & $-35.29 * *$ & $-42.86 * *$ & $7.32 * *$ & $-16.98 * *$ & $-35.29 * *$ \\
\hline MM 2008-8 X MM-306 & $-4.44 * *$ & $-4.44 * *$ & $-16.67 * *$ & $-20.93 * *$ & $-50.00 * *$ & $-55.84 * *$ & $31.71 * *$ & $1.89 * *$ & $-20.59 * *$ \\
\hline MM 2008-8 X MM-311 & $-20.00^{* *}$ & $-20.00 * *$ & $-30.23 * *$ & $-32.56 * *$ & $-57.35 * *$ & $-62.34 * *$ & $17.07 * *$ & $-9.43 * *$ & $-29.41 * *$ \\
\hline MM 2008-8 X MM-304 & $-6.67 * *$ & $-6.67^{* *}$ & $-18.60^{* *}$ & $16.28^{* *}$ & $-26.47 * *$ & $-35.06 * *$ & $26.83 * *$ & $-1.89 * *$ & $-23.53 * *$ \\
\hline MM-310 X IC-267375 & $-31.11 * *$ & $-31.11^{* *}$ & $-39.92 * *$ & $132.56^{* * *}$ & $47.06^{* *}$ & $29.87 * *$ & $36.59 * *$ & $5.66 * *$ & $-17.65^{* *}$ \\
\hline MM-310 X MM-303 & $-15.56 * *$ & $-15.56^{* *}$ & $-26.36^{* *}$ & $120.93 * *$ & $39.71 * *$ & $23.38 * *$ & $31.71 * *$ & $1.89 * *$ & $-20.59 * *$ \\
\hline MM-310 X MM-308 & $-20.00 * *$ & $-20.00 * *$ & $-30.23 * *$ & $69.77^{* *}$ & $7.35^{* *}$ & $-5.19^{* *}$ & 0.00 & $-22.64 * *$ & $-39.71^{* *}$ \\
\hline MM-310 X PS & $-6.67 * *$ & $-6.67^{* *}$ & $-18.60 * *$ & $79.07^{* *}$ & $13.24 * *$ & 0.00 & 0.00 & $-22.64 * *$ & $-39.71 * *$ \\
\hline MM-310 X MM-306 & $-16.44 * *$ & $-16.44 * *$ & $-27.13 * *$ & $69.77^{* * *}$ & $7.35^{* *}$ & $-5.19 * *$ & $24.39 * *$ & $-3.77 * *$ & $-25.00^{* *}$ \\
\hline MM-310 X MM-311 & $-9.78 * *$ & $-9.78 * *$ & $-21.32 * *$ & $97.67^{* *}$ & $25.00 * *$ & $10.39 * *$ & 0.00 & $-22.64 * *$ & $-39.71 * *$ \\
\hline MM-310 X MM-304 & $-11.11 * *$ & $-11.11^{* *}$ & $-22.48 * *$ & $93.02 * *$ & $22.06^{* *}$ & $7.79 * *$ & $29.27 * *$ & 0.00 & $-22.06 * *$ \\
\hline IC-267375 X MM-303 & $-8.89 * *$ & $-8.89^{* *}$ & $-20.54 * *$ & $44.19^{* *}$ & $-8.82 * *$ & $-19.48^{* *}$ & $21.95 * *$ & $-5.66 * *$ & $-26.47 * *$ \\
\hline IC-267375 X MM-308 & $-16.89 * *$ & $-16.89^{* *}$ & $-27.52^{* *}$ & $86.05^{* *}$ & $17.65^{* *}$ & $3.90^{* *}$ & $26.83 * *$ & $-1.89 * *$ & $-23.53 * *$ \\
\hline IC-267375 X PS & $-22.22 * *$ & $-22.22^{* *}$ & $-32.17 * *$ & $34.88^{* *}$ & $-14.71 * *$ & $-24.68 * *$ & $26.83 * *$ & $-1.89 * *$ & $-23.53 * *$ \\
\hline IC-267375 X MM-306 & $-15.56^{* * *}$ & $-15.56^{* *}$ & $-26.36 * *$ & $-2.33^{* *}$ & $-38.24 * *$ & $-45.45^{* *}$ & $12.20^{* *}$ & $-13.21^{* *}$ & $-32.35^{* *}$ \\
\hline IC-267375 X MM-311 & $-8.89 * *$ & $-8.89 * *$ & $-20.54 * *$ & $55.81^{* *}$ & $-1.47 * *$ & $-12.99 * *$ & $36.59 * *$ & $5.66^{* * *}$ & $-17.65^{* *}$ \\
\hline IC-267375 X MM-304 & $-17.78^{* *}$ & $-17.78^{* *}$ & $-28.29 * *$ & $11.63^{* *}$ & $-29.41 * *$ & $-37.66 * *$ & $4.88 * *$ & $-18.87 * *$ & $-36.76^{* *}$ \\
\hline MM-303 X MM-308 & $9.78 * *$ & $9.78^{* *}$ & $-4.26 * *$ & $58.14 * *$ & 0.00 & $-11.69 * *$ & $26.83 * *$ & $-1.89 * *$ & $-23.53 * *$ \\
\hline MM-303 X PS & $-15.56 * *$ & $-15.56^{* *}$ & $-26.36 * *$ & $109.30^{* * *}$ & $32.35 * *$ & $16.88^{* *}$ & $17.07 * *$ & $-9.43 * *$ & $-29.41 * *$ \\
\hline MM-303 X MM-306 & $13.33^{* *}$ & $13.33^{* *}$ & $-1.16 * *$ & $86.05^{* *}$ & $17.65^{* * *}$ & $3.90 * *$ & $39.02 * *$ & $7.55^{* * *}$ & $-16.18^{* *}$ \\
\hline MM-303 X MM-311 & $17.78^{* *}$ & $17.78^{* *}$ & $2.71^{* *}$ & $86.05^{* *}$ & $17.65^{* *}$ & $3.90^{* *}$ & $34.15^{* *}$ & $3.77 * *$ & $-19.12 * *$ \\
\hline MM-303 X MM-304 & $13.33 * *$ & $13.33 * *$ & $-1.16 * *$ & $79.07^{* *}$ & $13.24 * *$ & 0.00 & $43.90 * *$ & $11.32 * *$ & $-13.24 * *$ \\
\hline MM-308 X PS & $2.22 * *$ & $2.22 * *$ & $-10.85 * *$ & $37.21^{* * *}$ & $-13.24 * *$ & $-23.38 * *$ & $41.46 * *$ & $9.43 * *$ & $-14.71 * *$ \\
\hline MM-308 X MM-306 & $5.78 * *$ & $5.78^{* *}$ & $-7.75 * *$ & $39.53^{* *}$ & $-11.76^{* *}$ & $-22.08 * *$ & $7.32 * *$ & $-16.98 * *$ & $-35.29 * *$ \\
\hline MM-308 X MM-311 & $6.67 * *$ & $6.67 * *$ & $-6.98 * *$ & $34.88^{* * *}$ & $-14.71^{* *}$ & $-24.68^{* *}$ & $19.51^{* *}$ & $-7.55 * *$ & $-27.94 * *$ \\
\hline MM-308 X MM-304 & $8.89 * *$ & $8.89 * *$ & $-5.04 * *$ & $37.21^{* *}$ & $-13.24 * *$ & $-23.38 * *$ & $26.83 * *$ & $-1.89 * *$ & $-23.53 * *$ \\
\hline PSX MM-306 & $-6.67 * *$ & $-6.67 * *$ & $-18.60 * *$ & $86.05^{* *}$ & $17.65^{* *}$ & $3.90^{* *}$ & $36.59 * *$ & $5.66 * *$ & $-17.65^{* *}$ \\
\hline PS X MM-311 & $5.78 * *$ & $5.78^{* *}$ & $-7.75 * *$ & $51.16^{* *}$ & $-4.41 * *$ & $-15.58^{* *}$ & $29.27 * *$ & 0.00 & $-22.06^{* *}$ \\
\hline PS X MM-304 & $11.11 * *$ & $11.11 * *$ & $-3.10 * *$ & $67.44 * *$ & $5.88^{* *}$ & $-6.49 * *$ & $34.15^{* *}$ & $3.77 * *$ & $-19.12^{* *}$ \\
\hline MM-306 X MM-311 & $10.22 * *$ & $10.22 * *$ & $-3.88 * *$ & $44.19^{* *}$ & $-8.82 * *$ & $-19.48^{* *}$ & $34.15 * *$ & $3.77 * *$ & $-19.12 * *$ \\
\hline MM-306 X MM-304 & $17.78^{* *}$ & $17.78^{* *}$ & $2.71 * *$ & $48.84^{* *}$ & $-5.88 * *$ & $-16.88^{* *}$ & $34.15^{* *}$ & $3.77^{* *}$ & $-19.12^{* *}$ \\
\hline MM-311 X MM-304 & $5.33 * *$ & $5.33 * *$ & $-8.14 * *$ & $72.09 * *$ & $8.82 * *$ & $-3.90 * *$ & $46.34 * *$ & $13.21 * *$ & $-11.76^{* *}$ \\
\hline CD 5\% & & 1.01 & & & 0.52 & & & 0.28 & \\
\hline CD 1\% & & 1.33 & & & 0.68 & & & 0.36 & \\
\hline
\end{tabular}


Twelve hybrids, MS-5 $\times$ MM-306, MS-5 $\times$ MM-310, MS-5 $\times$ PS, MM-310 $\times$ IC-267375, MM-306 × MM-304, MM-310 × MM-303, MM-311 × MM-304, MM-308 × MM-311, MM-306 $\times$ MM-311, MM-303 $\times$ PS, MS-5 $\times$ MM-308, MS-5 $\times$ MM-311 have shown superior performance in respect to days to fruit maturity, fruit weight, TSS content, rind thickness, flesh thickness, fruit cavity area, ascorbic acid content, total yield per plot, acidity and reaction to fusarium wilt disease over the check Punjab Hybrid (Table 5). The cross combination MS-5 $\times$ MM-306 exhibited high heterosis for days to fruit maturity, fruit weight, TSS content, rind thickness, flesh thickness, fruit cavity area, ascorbic acid content, acidity and reaction to fusarium wilt disease over the check Punjab Hybrid. The cross combination MS-5 × MM-308 have displayed high heterosis for total yield per plot, days to fruit maturity, fruit weight rind thickness, flesh thickness, fruit cavity area, ascorbic acid content and acidity over the Punjab hybrid. The hybrid MM-311 × MM304 had high heterosis for rind thickness, fruit weight, TSS content, flesh thickness, fruit cavity area, ascorbic acid content and fruit shape index over Punjab Hybrid. Similarly the hybrid MS-5× MM-311 had high heterosis for fruit weight days to maturity, rind thickness, flesh thickness, $\beta$ - carotene content, acidity and fruit shape index over Punjab Hybrid. The cross combination MS-5 $\times$ PS exhibited high heterosis over Punjab hybrid for fruit weight, days to maturity, rind thickness, flesh thickness, fruit cavity area, acidity and flesh thickness. The hybrid MM-308 × MM-311 had high heterosis for TSS content, days to maturity, rind thickness, flesh thickness, fruit cavity area, ascorbic acid content and fruit shape index. The hybrid, MM-310 $\times$ IC267375 exploited significant heterosis for total yield per plot, rind thickness, flesh thickness, ascorbic acid content and acidity over the Punjab hybrid. The cross combination MS-5× MM-310 had high heterosis for fruit weight, TSS content along with rind thickness, fruit shape index, acidity and ascorbic acid content over Punjab hybrid. The hybrid MM-306 × MM-304 exhibited significant heterosis for fruit weight, TSS content, rind thickness, flesh thickness, fruit cavity area and acidity over check Punjab hybrid. The cross combination MM-306 × MM-311 revealed high heterosis for fruit weight, TSS content, rind thickness, flesh thickness, fruit cavity area, ascorbic acid content and reaction to fusarium wilt disease over Punjab hybrid. The hybrid, MM-303 × PS had high heterosis for TSS content, rind thickness, flesh thickness, acidity, fruit shape index and reaction to fusarium wilt disease over Punjab hybrid.

From the present investigation, it is inferred that five hybrids viz., MS-5 × MM 308, MS-5 $\times$ MM 306, MS-5 × MM-304, MM-308 × MM-306 and MM-306 $\times$ MM-304 were found promising and were significantly better and (or) statistically at par with the best standard checks for fruit yield and TSS content along with some other important attributes. The results of the present investigation were based on single location evaluation. Thus, the above five $F_{1}$ hybrids should be tested over multi-locations to make the results more reliable and of wider acceptability. The promising hybrids also have potential to give transgressive segregants in the early segregating generations. The transgressive segregants so generated can be utilized to develop superior inbred lines.

\section{References}

Anonymous, 2014. Package of Practices for Cultivation of Vegetables. $\mathrm{Pp}$ 2-4. Punjab Agricultural University, Ludhiana.

Banga, S.S. and Banga, S. K. (ed) (2000) Hybrid Cultivar Development. pp. 17- 
31 Narosa Publishing House, New Delhi

Choudhary, B.R., Dhaka, R. S. and Fageria, M. S. 2003. Heterosis for yield and yield related attributes in muskmelon. Indian J Genet 63: 91-92.

Choudhary, B.R., Fageria, M. S., Pandey, S. and Rai, M. 2006. Combining ability studies for economic attributes in muskmelon. Veg Sci 33: 185-87

Dhaliwal, M. S. and Lal, T. 1996. Genetics of some important characters using line $\mathrm{x}$ tester analysis in muskmelon. Indian $J$ Genet 56: 207-13.

Dhillon, B. S. 1998. Maize. In: S. S. Banga and S. K. Banga (ed) Hybrid Cultivar Development. Pp. 282-306. Narosa Publishing. House, New Delhi.

Ferreira, F. M., Ribeiro Junior, J. I., Pacheco, C. A. P., Silva, C. H. O. and Martins Filho, S. 2004. Genetic components of combining ability in a complete diallel. Crop Breeding and Applied Biotech 4: 338-43.

Griffing, B. 1956. Concept of general and specific combining abilities in relation to diallel cross system. Aust J Biol Univ 25: 149-52

Gurav, S. B., Wavhal, K. N. and Navale, P. A. 2000. Heterosis and combining ability on muskmelon. J Maharashtra Agric Univ 25: 149-52. (Original not seen.
Abstr. in Biological Abstracts, 14: Entry No. 20013067327, 2000)

Kalb, T. J. and Davis, D. W. 1984. Evaluation of combining ability, heterosis and genetic variance for fruit quality characteristics in bush muskmelon. $J$ Amer Soc Hort Sci 109: 411-15

Kesavan, P. K. and More, T. A. 1991. Combining ability studies in muskmelon. Veg Sci 18: 146-50.

Kitroongruang, N., Poo-Swang, W., and Tokumasu, S. 1992. Evaluation of combining ability, heterosis and genetic variance of plant growth and fruit quality in muskmelon. Scientia Hort 50: 79-87.

Liou, L. J., Li, S. M. and Li, S. Q. 1995. Study of breed cross between the thin skinned muskmelon (spp. melo): expression of $F_{1}$ hybrids and analysis of combining ability for the parents. Acta Hort 402: 48-51.

Munshi, A. D. and Verma, V. K. 1997.Studies of heterosis in muskmelon. Veg Sci 24: 103-06.

Munshi, A. D. and Verma, V. K. 1999. Combining ability of muskmelon. Indian J Agric Sci 69: 214-16

Vashisht, V. K., Sehgal, G., Lal, T. and Gaikwad, A. K. 2010. Combining ability for yield and yield attributing traits in muskmelon. Crop Improv 37: $36-4$

\section{How to cite this article:}

Singh Varinder and Vashisht, V.K. 2018. Heterosis and Combining Ability for Yield in Muskmelon (Cucumis melo L.). Int.J.Curr.Microbiol.App.Sci. 7(08): 2996-3006. doi: https://doi.org/10.20546/ijcmas.2018.708.318 